\title{
Prevalencia de síntomas no motores en pacientes con enfermedad de parkinson: una revision.
}

\author{
Prevalence of non-motor symptoms in patients with parkinson's \\ disease: a review.
}

Cuba-Antezana Analii, a Anicama-Hernández Ángel ${ }^{1, a}$

1. Medico neurólogo

a. Hospital Regional de Ica

\section{Correspondencia:}

Anali Cuba-Antezana.

Número de celular: 997600520

Correo Electrónico:

anamire_16@hotmail.com

Domicilio: Calle Casuarinas

Residencial la angostura, Ica, Perú.

Contribuciones De Autoría:

ACA, AAH: Contribuciones en la

concepción y diseño del manuscri-

to, recolección, análisis e

interpretación de los datos,

redacción y revisión crítica del

contenido del manuscrito y

aprobación final del artículo.

Conflicto De Intereses: No

declarados.

Financiamiento: Autofinanciado.

\section{Como Citar}

Cuba-Antezana Anali. Prevalencia de síntomas no motores en pacientes con enfermedad de parkinson: una revision Rev méd panacea 2018; (3): 101-106

Recibido: 26 - 09 - 2018

Aceptado: 30 - $11-2018$

Publicado: 28 - 12 - 2018

\section{RESUMEN}

Introducción: La enfermedad de Parkinson constituye una de las enfermedades neurodegenerativas más prevalentes en el mundo y en nuestro país, afectando el 1 al $3 \%$ de la población, debido a su carácter progresivo afecta de forma importante la calidad de vida de quienes la padecen. Dentro de la enfermedad existen los síntomas no motores los cuales han ganado relevancia en los últimos años debido a su elevada prevalencia que se incrementa conforme la enfermedad progresa y un impacto negativo sobre la calidad de vida de los pacientes que puede ser mayor que el de las manifestaciones motoras (1). Objetivo: Realizar una búsqueda bibliográfica para determinar la prevalencia de síntomas no motores en pacientes con Enfermedad de Parkinson. Materiales y métodos: : Es un estudio descriptivo en donde se realiza la búsqueda bibliográfica en la base de datos de Pubmed, Medline y la Academia Americana de Neurología. Discusión: los síntomas no motores en la enfermedad de Parkinson incluyen síntomas vegetativos, neuropsiquiátricos, anosmia, trastornos del sueño, anomalías oculares entre las más frecuentes que esta presentes en un 80 a 90\% según la literatura. Según esta revisión se encuentra como síntoma no motor más frecuente la depresión, habiendo diferencias entre hombres y mujeres, y si toman o no medicación antiparkinsoniana. Conclusiones: Los síntomas no motores en pacientes con Enfermedad de Parkinson constituyen un aspecto importante de la enfermedad, que incluso preceden a los síntomas motores por lo cual deben ser detectados tempranamente para un manejo oportuno y multidisciplinario.

Palabras clave: enfermedad de Parkinson, prevalencia, síntomas no motores. (Fuente DeCS)

\begin{abstract}
Introduction: Parkinson's disease is one of the most prevalent neurodegenerative diseases in the world and in our country, affecting 1 to $3 \%$ of the population, due to its progressive nature significantly affects the quality of life of those who suffer from it. Within the disease there are non-motor symptoms which have gained relevance in recent years due to its high prevalence that increases as the disease progresses and a negative impact on the quality of life of patients that may be greater than that of the motor manifestations (1). Objective: To conduct a literature search to determine the prevalence of non-motor symptoms in patients with Parkinson's disease. Materials and methods: This is a descriptive study in which the bibliographic search is carried out in the Pubmed, Medline and American Academy of Neurology databases. Discussion: non-motor symptoms in Parkinson's disease include vegetative symptoms, neuropsychiatric symptoms, anosmia, sleep disorders, ocular abnormalities among the most frequent, which are present in 80 to $90 \%$ according to the literature. According to this review, depression is the most common non-motor symptom, there are differences between men and women, and whether or not they take antiparkinson medication. Conclusions: Non-motor symptoms in patients with Parkinson's disease are an important aspect of the disease, which even precede motor symptoms, which is why they should be detected early for timely and multidisciplinary management.
\end{abstract}

Keywords: Parkinson's disease, prevalence, non-motor symptoms. (Fuente MesH). 


\section{INTRODUCCIÓN}

La enfermedad de Parkinson (EP) es una enfermedad neurodegenerativa clínicamente caracterizada por bradicinesia, temblor en reposo y rigidez. La pérdida de neuronas dopaminérgicas en la sustancia negra se correlaciona con las manifestaciones motores de la enfermedad de Parkinson. La progresión de estos síntomas cardinales es insidiosa y el diagnóstico de la enfermedad es hecho comúnmente después de la aparición de las manifestaciones motoras.

Por otra parte, los síntomas no motores (SNM) en la EP son frecuentes en todos los estadios de la enfermedad, y representan frecuentemente las principales quejas y molestias de los pacientes, de manera que estos influyen en la calidad de vida del paciente. Los SNM en la EP son heterogéneos e incluyen disfunción cognitiva (demencia), trastorno psiquiátrico (desde depresión y ansiedad hasta alucinaciones y psicosis), trastornos del sueño (somnolencia excesiva de día y trastorno del comportamiento de la fase de sueño MOR) y disfunción autonómica (estreñimiento, hipotensión postural, disfunción eréctil y trastornos genitourinarios (1).

Aunque las características no motoras en la enfermedad de Parkinson (EP) son comunes, estos síntomas a menudo no son bien reconocidos en la práctica clínica. Varios estudios previos han abordado la importancia de los síntomas no motores como causa de discapacidad y deterioro de la calidad de vida, y como una razón importante para el ingreso a la atención institucional.

El Cuestionario de Síntomas No Motrices (NMSQuest) se desarrolló para abordar la necesidad de un instrumento cuantitativo y validado para medir el NMS en EP. El NMSQuest es una herramienta de detección autocompletada diseñada para evaluar la presencia de NMS en pacientes con EP. Está compuesto por 30 elementos agrupados según nueve dominios.

El objetivo de este artículo es realizar una revisión actualizada sobre la prevalencia de síntomas no motores y dentro de ellos cual es el más frecuente dentro de los pacientes con enfermedad de Parkinson, y si hubiera o no diferencias entre hombres y mujeres y si toman o no mediación antiparkinsoniana.

\section{MATERIALES Y MÉTODOS}

La búsqueda bibliografía se ha realizado en Pubmed, Medline y la Academia Americana de Neurología. Como criterios de selección se tomó en cuenta información publicada en dichas fuentes dada la confiabilidad de las mismas, con una antigüedad no mayor de 5 años, tanto en idioma español como inglés.

\section{LA ENFERMEDAD DE PARKINSON PREVALENCIA}

La enfermedad de Parkinson (EP) tiene una presentación clínica relativamente estereotipada que ahora lleva el nombre de este médico de principios del siglo XIX. La EP es uno de los trastornos neurológicos más comunes en todo el mundo. La incidencia anual es de 8-18 casos nuevos por 100.000 habitantes, con el pico en la sexta década de la vida. La prevalencia se incrementa con la edad: 0,6/100 casos entre los 65 y los 69 años y 5/100 casos entre los 85 y los 89 años (2).

\section{DEFINICIÓN}

La enfermedad de Parkinson, que se definió sobre bases clínico patológicas, se caracteriza por la coexistencia de un síndrome rígido-acinético y temblor de reposo, con una degeneración de la pars compacta de la sustancia negra y la presencia de inclusiones neuronales denominadas cuerpos de Lewy (CL) compuestos, principalmente, de alfa-sinucleína anormal (3).

\section{ETIOLOGÍA}

A pesar de la investigación intensiva, la etiología precisa de la DP sigue siendo difícil de alcanzar. Una conceptualización es que una toxina ambiental desconocida actúa sobre individuos genéticamente susceptibles. El principal enlace entre la EP y una toxina ambiental es el químico MPTP (1-metil-4-fenil-1,2,3,6-tetrahidropiridina) (4). La mayoría de los casos de EP son esporádicos, y sólo un 8 al $10 \%$ presenta un patrón mendeliano de transmisión hereditaria, pero la importancia de la influencia genética, incluso en los casos aparentemente esporádicos, está creciendo muy rápidamente. Se han descubierto varios loci genéticos como el GBA, PARK 1-15, PARKIN, LRRK2, PINK1 Y DJ1 entre otros.

\section{ANATOMÍA PATOLÓGICA}

Las lesiones histológicas combinan la pérdida de neuronas y la acumulación de cuerpos de Lewy (CL). En algunas estructuras circunscritas, como en la $\mathrm{SN}$, el núcleo motor dorsal del vago, el locus coeruleus o el núcleo pedunculo-pontino es fácil apreciar ambas lesiones, mientras que en otras es difícil demostrar la pérdida neuronal (p. ej., en la corteza). En los núcleos cuyas neuronas contienen neuromelanina se observa la liberación del pigmento que aparece fagocitado por microglía (5).

\section{FISIOPATOLOGÍA}

La SN compacta ventral proyecta terminaciones dopaminérgicas al putamen, mientras que la porción dorsolateral lo hace al caudado. El área tegmental se proyecta hacia la región frontobasal y septal (núcleo acumbens). Estos núcleos proporcionan la práctica totalidad de la síntesis fisiológica y de las proyecciones subcorticales de DA. De ahí que su lesión produzca un déficit de DA tan importante en el sistema nervioso central (SNC). El déficit dopaminérgico estriatal es el factor más importante para la aparición de los síntomas y signos motores de la enfermedad. Otros sistemas de neurotransmisores también se encuentran alterados (descenso de GABA, serotonina y catecolaminas), pero su significado es incierto. En los pacientes con deterioro cognitivo se encuentra un déficit colinérgico sobre cuya 
base se ha propuesto el tratamiento con anticolinesterásicos (6).

\section{CUADRO CLÍNICO}

\section{SÍNTOMAS MOTORES}

Según los criterios del Banco de Cerebros del Reino Unido esto se fundamenta en 4 signos clínicos principales:

- Acinesia o bradicinesia: la lentitud para efectuar movimientos voluntarios o automáticos. La mayoría de los enfermos refiere la acinesia como «falta de firmeza». - Rigidez: es un estado continuo de tensión y firmeza muscular que dificulta el desplazamiento de las articulaciones. - Temblor: típico es de reposo, con una frecuencia de 4-6 Hz, aumenta con la concentración (recitar los meses hacia atrás) y con el estrés de sentirse observado, y desaparece con el sueño.

- Trastornos de la postura: No suelen ser evidentes en las fases precoces de la enfermedad, sobre todo si es unilateral (7).

\section{SÍNTOMAS NO MOTORES}

El reconocimiento de los síntomas no motores ha ganado relevancia en los últimos años debido a su elevada prevalencia que se incrementa conforme la enfermedad progresa y un impacto negativo sobre la calidad de vida de los pacientes que puede ser mayor que el de las manifestaciones motoras. La prevalencia se encuentra entre el 80 al $90 \%$ en estos pacientes (8). Dentro de las que se describen son las siguientes:

- Alteraciones vegetativas: La disfunción autonómica en la EP es común y prácticamente todos los aspectos de la función autonómica pueden verse afectados. Así tenemos:

* La hipotensión ortostática es el aspecto más ampliamente reconocido de la disfunción cardiovascular en la EP.

* La gastroparesia con sensación de saciedad precoz, disminución del apetito, distensión abdominal, distensión abdominal, náuseas, vómitos y pérdida progresiva de peso.

* La hiperactividad del detrusor es la anomalía urodinámica más frecuente, que produce urgencia urinaria, frecuencia, nicturia e incontinencia del tipo de urgencia.

* La disfunción sexual es muy común tanto en hombres como en mujeres con EP

* Problemas de la sudoración: hiperhidrosis episódica.

* La sialorrea se debe a la acinesia de la deglución.

- Anosmia: La pérdida del olfato rara vez es referida de manera espontánea por el paciente.

- Anomalías oculares: Destacan el reflejo glabelar inagotable, el cierre involuntario de los párpados bien por blefaroespasmo o bien por apraxia palpebral (o por ambos), y las dificultades para la convergencia y la mirada conjugada vertical hacia arriba.

- Alteraciones del sueño: El sueño tiende a estar fragmentado, con reducción sobre todo del sueño No REM y del sueño REM como el trastorno de piernas inquietas y movimiento periódico de piernas.

- Trastorno cognitivo: como la lentitud de los procesos mentales y de la toma de decisiones, perseveración, disminución de la fluencia verbal, alteración de la memoria de trabajo o secuenciación cognitiva, y también de las tareas visuoespaciales.

- Trastornos del estado de ánimo: Los síntomas del estado de ánimo son frecuentes en la EP, con depresión y ansiedad es más comúnmente encontrado.

- Trastornos psiquiátricos: tales como la psicosis la cual es frecuente, afectando a un tercio de los pacientes con EP tratados con terapia dopaminérgica crónica y más del $50 \%$ en estudios longitudinales de 10 años o más. Así también las alucinaciones visuales que son muy frecuentes en los pacientes avanzados y especialmente si evolucionan hacia la demencia de la que no rara vez son el primer síntoma. Se agravan por los fármacos dopaminérgicos, sobre todo por los agonistas.

- El Trastorno del control de impulsos en la cual se incluyen comportamientos inadecuados de los pacientes que pueden ser perjudiciales, e incluso autodestiructivos, como por ejemplo la tendencia compulsiva al juego, hiperfagia compulsiva, hipererotismo y promiscuidad, tricotilomanía y cleptomanía (9).

\section{VALORACION DE SINTOMAS NO MOTORES} CUESTIONARIO PARA SINTOMAS NO MOTORES (SNM) El cuestionario NMS es un cuestionario de 30 ítems con respuestas como "sí", "no" y "no sé" para cada ítem (tabla 1)(9), los cuales son contestadas por el paciente o formuladas por el medico mediante una escala tipo likert. 
Tabla 1: Cuestionario de Síntomas no Motores en Enfermedad de Parkinson (PD SNM Quest)

\begin{tabular}{|c|c|c|c|}
\hline SINTOMAS NO MOTORES & SI & No & NO SABE \\
\hline Babeo durante el día & & & \\
\hline Pérdida o alteración en la percepción de & & & \\
\hline $\begin{array}{l}\text { Dificultad para tragar comida o bebidas, o } \\
\text { tendencia a atragantarse }\end{array}$ & & & \\
\hline Vómitos o nauseas & & & \\
\hline $\begin{array}{l}\text { Estreñimiento (realizar deposiciones menos de } 3 \\
\text { veces a la semana) o tener que hacer esfuerzos } \\
\text { para ello }\end{array}$ & & & \\
\hline Incontinencia fecal (se escapan las heces) & & & \\
\hline $\begin{array}{l}\text { Sensación de no haber vaciado por completo el } \\
\text { vientre después de ir al servicio }\end{array}$ & & & \\
\hline $\begin{array}{l}\text { Sensación de tener que orinar urgentemente que le } \\
\text { obliga a ir rápidamente al servicio }\end{array}$ & & & \\
\hline $\begin{array}{l}\text { Necesidad de levantarse habitualmente por la } \\
\text { noche a orinar }\end{array}$ & & & \\
\hline $\begin{array}{l}\text { Dolores sin causa aparente (no debidos a otras } \\
\text { enfermedades, como la artrosis) }\end{array}$ & & & \\
\hline $\begin{array}{l}\text { Cambio de peso sin causa aparente (no debido a } \\
\text { un régimen o dieta) }\end{array}$ & & & \\
\hline $\begin{array}{l}\text { Problemas para recordar cosas que han pasado } \\
\text { recientemente o dificultad para acordarse de cosas } \\
\text { que tenía que hacer }\end{array}$ & & & \\
\hline $\begin{array}{l}\text { Pérdida de interés en lo que pasa a su alrededor o } \\
\text { en realizar sus actividades }\end{array}$ & & & \\
\hline $\begin{array}{l}\text { Ver u oír cosas que sabe o que otras personas le } \\
\text { dicen que no están ahí }\end{array}$ & & & \\
\hline Dificultad para concentrarse o mantener la atenciór & & & \\
\hline Sentirse triste, bajo/a de ánimo o decaído & & & \\
\hline Sentimientos de ansiedad, miedo o pánico & & & \\
\hline Pérdida o aumento del interés por el sexo & & & \\
\hline Dificultades en la relación sexual cuando lo intenta & & & \\
\hline $\begin{array}{l}\text { Sensación de mareo o debilidad al ponerse de pie } \\
\text { después de haber estado sentado o tumbado }\end{array}$ & & & \\
\hline Caídas & & & \\
\hline $\begin{array}{l}\text { Dificultad para mantenerse despierto/a mientras } \\
\text { realiza actividades como trabajar, conducir o } \\
\text { comer }\end{array}$ & & & \\
\hline $\begin{array}{l}\text { Dificultad para quedarse o mantenerse dormido } \\
\text { por la noche }\end{array}$ & & & \\
\hline Sueños intensos, vívidos o pesadillas & & & \\
\hline $\begin{array}{l}\text { Hablar o moverse durante el sueño como si lo } \\
\text { estuviera viviendo }\end{array}$ & & & \\
\hline $\begin{array}{l}\text { Sensaciones desagradables en las piernas por la } \\
\text { noche o cuando está descansando, y sensación de } \\
\text { que necesita moverlas }\end{array}$ & & & \\
\hline Hinchazón en las piernas & & & \\
\hline Sudoración excesiva & & & \\
\hline Visión doble & & & \\
\hline $\begin{array}{l}\text { Creer que le pasan cosas que otras personas le } \\
\text { dicen que no son verdad }\end{array}$ & & & \\
\hline
\end{tabular}


Se han realizado diversas revisiones en la relación al síntoma motor más prevalente como en el trabajo de de Martínez-Ramírez D. et al. (10) titulado Relación entre el género y los síntomas no motores en pacientes con enfermedad de Parkinson publicado en el año 2013 en la revista de neurociencias de México cuyo objetivo fue determinar si existe asociación entre género y síntomas no motores. Los síntomas más comunes en mujeres ( $p$ <0.05) fueron: vómito/náusea, estreñimiento, dolor inexplicable, memoria, alucinaciones, tristeza, ansiedad, mareo, caídas e insomnio. El síntoma más común en hombres fue dificultad sexual. Conclusiones: se encontraron diferencias significativas entre hombres y mujeres en la prevalencia de algunos síntomas no motores. Así también tenemos el estudio de Isais-Millán S. et al (11) titulado Prevalencia de trastornos neuropsiquiátricos en pacientes con enfermedad de Parkinson (EP) no tratados publicado en el año 2016 en la revista Gaceta de México. Tuvo como conclusión que los trastornos neuropsiquiátricos son frecuentes en pacientes con EP temprana sin tratamiento dopaminérgico.

Mientras que en el Perú solo se ha publicado un trabajo al respecto como el de Carlos Cosentino y col (12) con el título de Frequency of non-motor symptoms in Peruvian patients with Parkinson's disease publicado en el 2013, tuvo como objetivo determinar la prevalencia de los síntomas no motores de trescientos pacientes ambulatorios con enfermedad de Parkinson. La depresión de dominio obtuvo el mayor número de respuestas "positivas" mientras la incontinencia urinaria y la ansiedad / trastornos de memoria fueron segundo y tercero más frecuente, respectivamente.

\section{TRATAMIENTO DE LA ENFERMEDAD DE PARKINSON}

La variedad de tratamientos farmacológicos $y$ quirúrgicos disponibles para el tratamiento de la enfermedad de Parkinson (EP) idiopática (o del cuerpo de Lewy) es más amplia que para cualquier otra enfermedad degenerativa del sistema nervioso central. El manejo de pacientes individuales requiere una cuidadosa consideración de una serie de factores, que incluyen los síntomas y signos del paciente, la edad, el estadio de la enfermedad, el grado de discapacidad funcional y el nivel de actividad física y productividad. El tratamiento se puede dividir en terapia farmacológica, no farmacológica y quirúrgica.

El tratamiento farmacológico de la EP puede dividirse aún más en terapia neuroprotectora y sintomática. En la práctica, casi todos los tratamientos disponibles son de naturaleza sintomática y no parecen ralentizar o revertir el curso natural de la enfermedad. Sin embargo, varios posibles agentes neuroprotectores para la EP han demostrado ser prometedores en animales y / o humanos y están siendo investigados.

\section{Terapia sintomática}

La decisión de iniciar un tratamiento médico sintomático en pacientes con EP está determinada por el grado de deterioro funcional del paciente.

Los principales medicamentos disponibles para el tratamiento de los síntomas motores de PD incluyen:

1.- Levodopa

2.- Agonistas de dopamina

3.- Inhibidores de la monoaminooxidasa (MAO) B

4.- Agentes anticolinérgicos

5.- Amantadine

6.- Inhibidores de la Catecol-O-metiltransferasa (COMT) (13).

\section{DISCUSIÓN}

La Enfermedad de Parkinson constituye una de las enfermedades neurodegenerativas más frecuentes. Si bien es cierto los síntomas motores como la bradicinesia, el temblor, inestabilidad postural y la rigidez constituyen los síntomas cardinales de la enfermedad siendo el motivo por el cual los pacientes acuden a consulta médica, siendo la mayoría de los tratamientos avocados a dichos síntomas, la literatura reporta que la frecuencia de síntomas no motores constituyen un punto importante en estos pacientes que suele aparecer en etapas precoces de la enfermedad, incluso antes de los síntomas motores, los cuales no son reconocidos muchas veces por el paciente o el médico.

Es así que los síntomas no motores adquieren gran relevancia porque afectan directamente la calidad de vida de los pacientes con enfermedad de Parkinson, lo que hace necesario una identificación precoz y tratamiento específicos los cuales tienen que ser abordados por el médico, en este neurólogo.

De acuerdo a la literatura la prevalencia de síntomas no motores puede llegar hasta un 80 a 90\%, la mayoría de autores coinciden que la depresión es el síntomas no motor más prevalente, sin embargo, existen diferencias entre hombres y mujeres, siendo en los primeros más frecuente la disfunción sexual y en mujeres alteraciones gastrointestinales, así mismo en quienes toman o no medicación antiparkinsoniana, siendo los trastornos neuropsiquiatricos prevalentes en estos últimos.

\section{CONCLUSIONES}

La Enfermedad de Parkinson condiciona la calidad de vida de las personas que la sufren y se está convirtiendo en un problema de salud pública progresivamente mayor debido a su elevada frecuencia. Así mismo los síntomas no motores, que incluso pueden preceder a los síntomas motores constituyen un aspecto importante de la enfermedad que deberían ser identificados en la primera consulta para un manejo integral con la finalidad de mejorar la calidad de vida de estos pacientes. 


\section{REFERENCIAS BIBLIOGRÁFICAS}

1. Martinez-Fernandez R. et al. Actualización en la enfermedad de Parkinson. Rev. Med. Clin. Condes 2016; 27(3) 363-379.

2. J. Kulisevskya, M.R. Luquin et al. Enfermedad de Parkinson avanzada. Características clínicas y tratamiento parte I. Neurologia 2013, 1-19.

3. Zarranz. Enfermedades caracterizadas por movimientos anormales (enfermedades extrapiramidales o de los ganglios basales). Quinta edición, 2013.

4. Royden Jones et al. Netter's Neurology. Elsevier. 2013. Pag 280-291.

5. Jankovic J. et al. Etiology and pathogenesis of Parkinson disease. En uptodate 2017

6. J. Kulisevskya, M.R. Luquin et al. Enfermedad de Parkinson avanzada. Características clínicas y tratamiento parte II. Neurología 2013, 1-26.

7. Pfeiffer R. Non-motor symptoms in Parkinson's disease, Elsevier Parkinsonism and Related Disorders (2015) 1-4
8. Goldman et al. Neuropsychiatric Issues in Parkinson Disease. American Academy of Neurology, 2016.

9. Hess C. et all, Diagnosing Parkinson Disease. En revista Continuum 2016;22(4):1047-1063

10. Martínez-Ramírez D. et al. Relación entre el género y los síntomas no motores en pacientes con enfermedad de Parkinson. Arch Neurocien (Mex) Vol. 18, No. 3: 138-141; 2013.

11. Isais-Millán S. et al. Prevalencia de trastornos neuropsiquiátricos en pacientes con enfermedad de Parkinson (EP) no tratados. En Gac Med Mex. 2016;152:357-63

12. Cosentino C, Nuñez Y, Torres L. Frequency of non-motor symptoms in Peruvian patients with Parkinson's disease. Arq. Neuro-Psiquiatr. vol.71 no.4 São Paulo Apr. 2013.

13. Tarsy D. Pharmacologic treatment of Parkinson disease. En uptodate 2017.

\section{Las ediciones anteriores de revista médica PANACEA están disponibles en:}

\section{www.revpanacea.unica.edu.pe}

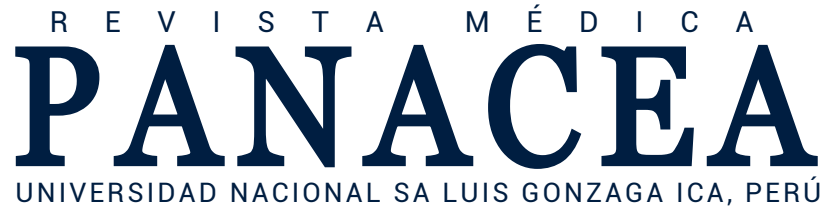

